\title{
A liquid biopsy signature predicts treatment response to fluoropyrimidine plus platinum therapy in patients with metastatic or unresectable gastric cancer: implications for precision oncology
}

\author{
In-Seob Lee ${ }^{1,2}$, Zhongxu Zhu ${ }^{3}$, Jeeyun Lee ${ }^{4}$, Joon Oh Park ${ }^{4}$, Xiwei Wu ${ }^{5}$, Tiffany Ong ${ }^{6}$, Sierra Min Li ${ }^{6}$, Xin Wang ${ }^{3}$, \\ Joseph Chao ${ }^{6^{*}+}$ and Ajay Goel ${ }^{1,7^{*}+}$
}

Patients with initially metastatic and/or locally advanced but unresectable gastric cancer (mGC) typically receive systemic chemotherapy based on a combination of fluoropyrimidine and platinum but prognosis in this group remains unsatisfactory [1-6]. Failure to proper transition of patients to subsequent therapies after disease progression on first-line treatment can risk exposing patients to worsening performance status, an upsurge of tumor burden, and exacerbation of toxicity due to the ineffective therapy. Therefore, pretreatment prediction of response to first-line chemotherapy is highly important for guiding patients to an optimal therapeutic approach and avoiding unnecessary toxicity.

Recent clinical analyses have consistently demonstrated that chemotherapy efficacy is associated with molecular subtyping of gastric cancer $(\mathrm{GC})[7,8]$. These data are of significant value as they suggest that optimal chemotherapy should be varied depending

\footnotetext{
*Correspondence: jchao@coh.org; ajgoel@coh.org

† Joseph Chao and Ajay Goel contributed equally to this work.

${ }^{6}$ Department of Medical Oncology and Therapeutics Research, City

of Hope Comprehensive Cancer Center, 1500 E. Duarte Road, Duarte, CA

91010, USA

${ }^{7}$ City of Hope Comprehensive Cancer Center, Duarte, CA, USA

Full list of author information is available at the end of the article
}

on tumor biologic characteristics. However, repeated access to obtain adequate tumor specimens for molecular profiling is limited by the invasiveness of endoscopic or percutaneous biopsy procedures and issues of tumor heterogeneity. Collectively, these observations highlight the unmet need for developing a blood-based biomarker to predict chemo-responsiveness in patients with mGC.

Herein, we conducted systematic, genome-wide expression profiling using small RNA sequencing in serum specimens from patients diagnosed with mGC, followed by bioinformatic approaches to identify a panel of miRNAs that could robustly discriminate responders from non-responders treated with a fluoropyrimidine and platinum doublet regimen. Then, we developed a response-prediction model, which we successfully validated in an independent clinical cohort. This systematic and comprehensive biomarker discovery and validation effort enabled us to identify patients who would benefit from standard chemotherapy, which may lead to more precise clinical decision-making strategies and improve treatment outcomes for patients with GC. 


\section{Results and discussion}

Genome-wide expression profiling identifies a novel 9-miRNA panel for predicting response to systemic chemotherapy

We analyzed sequencing-based miRNA expression profiling data from patients with mGC. Blood samples were prospectively collected from patients visiting the City of Hope Comprehensive Cancer Center, Duarte, CA, US before the commencement of chemotherapy. The data were from 8 responders (time to progression [TTP] greater than 6 months) and 4 non-responders (TTP less than 6 months), all of whom were treated with fluorouracil, oxaliplatin, and folinic acid (FOLFOX). Among 530 candidate miRNAs (after exclusion of miRNAs with a mean CPM [counts per million] less than 1), we identified 21 miRNAs that were differentially expressed between responders vs. non-responders $(P<0.01$; absolute log2 fold change $>2)$. Thereafter, we used an average expression $>7$ and an area under curve $($ AUC) $>0.8$ for individual miRNA candidates that discriminated responders from non-responders to identify a panel of 9 miRNAs: miR-30a-5p, miR-144-3p,
miR-192-5p, miR-451a, miR-619-5p, miR-625-5p, miR3168, miR-6873-3p, and miR-7157-5p. All 9 miRNAs showed high statistical significance (Fig. 1A); five miRNAs were upregulated and four were downregulated in the responder group. All miRNAs were accompanied by high average expression after differential expression analysis (Fig. 1B). Principal component analysis illustrated separate clusters for the responder and nonresponder groups (Fig. $1 \mathrm{C}$ ).

Next, we developed a logistic regression model to predict chemo-responsiveness in patients with $\mathrm{mGC}$, which yielded an AUC value of 1.00. Using a response probability score derived from the logistic regression model, we developed a heatmap of the cluster analysis for miRNA expression in relation to treatment response, which showed a separated clustering of expression between the two groups (Fig. 1D). The ability of the miRNA panel to identify the responder group is illustrated in the waterfall plot in Fig. 1E. Taken together, our biomarker discovery effort utilizing rigorous bioinformatic modules allowed us to successfully identify a 9-miRNA panel for prediction of chemo-responsiveness in patients with mGC.
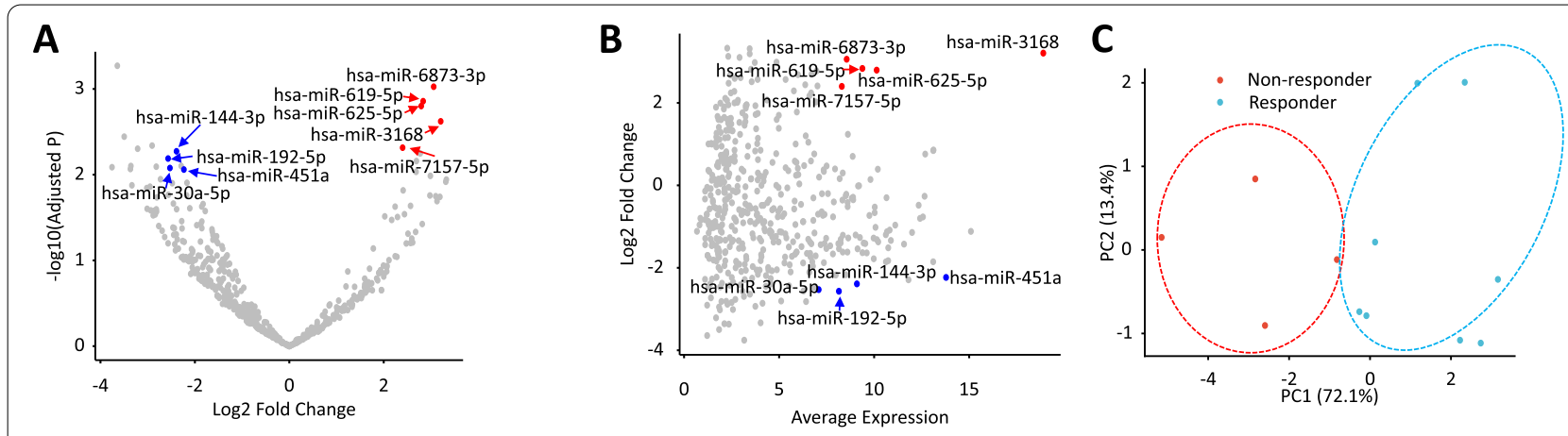

D



E

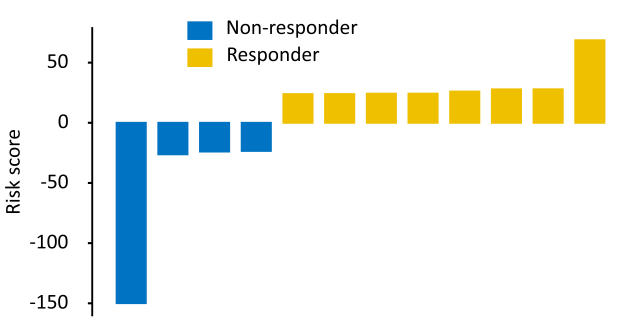

Fig. 1 A 9-miRNA panel discovered using small RNA sequencing data identifies patients responsive to systemic chemotherapy. A A volcano plot illustrates 9 miRNAs discovered from several bioinformatic approaches in sera from 12 patients with mGC. Among 21 miRNA candidates that were differentially expressed in responders and non-responders, logistic regression and average expression analyses reduced them to a 9-miRNA panel. B A volcano plot depicts differential expression of all 9 miRNAs after average expression analysis; 5 candidates were upregulated (red) and 4 were downregulated (blue) in responders vs, non-responders. C A principal component analysis shows separate clusters for responder and non-responder groups. D A heatmap illustrates clustering of miRNA expression based on response to systemic chemotherapy and estimated probability of chemo-responsiveness from the logistic regression analysis. E A waterfall plot demonstrates the ability of the 9-miRNA panel to discriminate responders from non-responders 


\section{Clinical validation leads to development of a formula} for predicting response to first-line chemotherapy

We next validated the 9-miRNA biomarker panel and developed a response-prediction formula by applying an optimized 2-miRNA biomarker panel consisting of miR-30a-5p and miR-192-5p to a clinical cohort of 29 patients with $\mathrm{mGC}$ treated with capecitabine and oxaliplatin (CapeOX) at Samsung Medical Center, Seoul, Korea. Response rate was evaluated using the Response Evaluation Criteria in Solid Tumors (RECIST) criteria version 1.1 [9].

There were 15 responders ( 1 case of complete response and 14 cases of partial response) and 14 non-responders (10 cases of stable disease and 4 cases of progressive disease). Their median age was 58 years and there were 17 males and 12 females. Median progression-free survival (PFS) and overall survival (OS) for this cohort were 4.1 and 16.1 months, respectively. There was no difference in gender, tumor location, Lauren classification, type of second-line treatment, or mean OS between responders and non-responders, but mean PFS was significantly longer in the responder group (9.1 vs. 2.7 months, $P=0.007$ ).

For biomarker validation, we performed RT-qPCR assays for each of the 9 miRNAs to measure expression in all samples. From these data, we developed a 2-miRNAbased prediction formula using logistic regression analysis through a backward elimination method, which exhibited an AUC of 0.79 in discriminating responders from non-responders (95\% CI: 0.60-0.92, sensitivity $60.0 \%$, specificity $92.9 \%, P=0.021$; Fig. 2 A). A waterfall plot illustrates the ability of our 2-miRNA biomarker panel to identify responders (Fig. 2B).

Next, we assessed the ability of the biomarker panel to identify only patients with progressive disease, in whom treatment intensification or altered chemotherapeutic drugs should be considered instead of doublet regimen. Our biomarker panel successfully identified patients who were expected to have the worst prognosis, with a corresponding AUC of 0.91 (95\% CI: 0.74-0.98, sensitivity: $84.0 \%$, specificity: $100 \%, P=0.008$; Fig. $2 \mathrm{C}$ ). We also verified that the response score was significantly different between two groups $(P=0.006)$ (Fig. 2D).

\section{The 2-miRNA biomarker panel robustly stratifies prognosis} of patients with $\mathrm{mGC}$

We dichotomized patients into groups with high- or lowprobability of chemo-responsiveness, according to cutoff

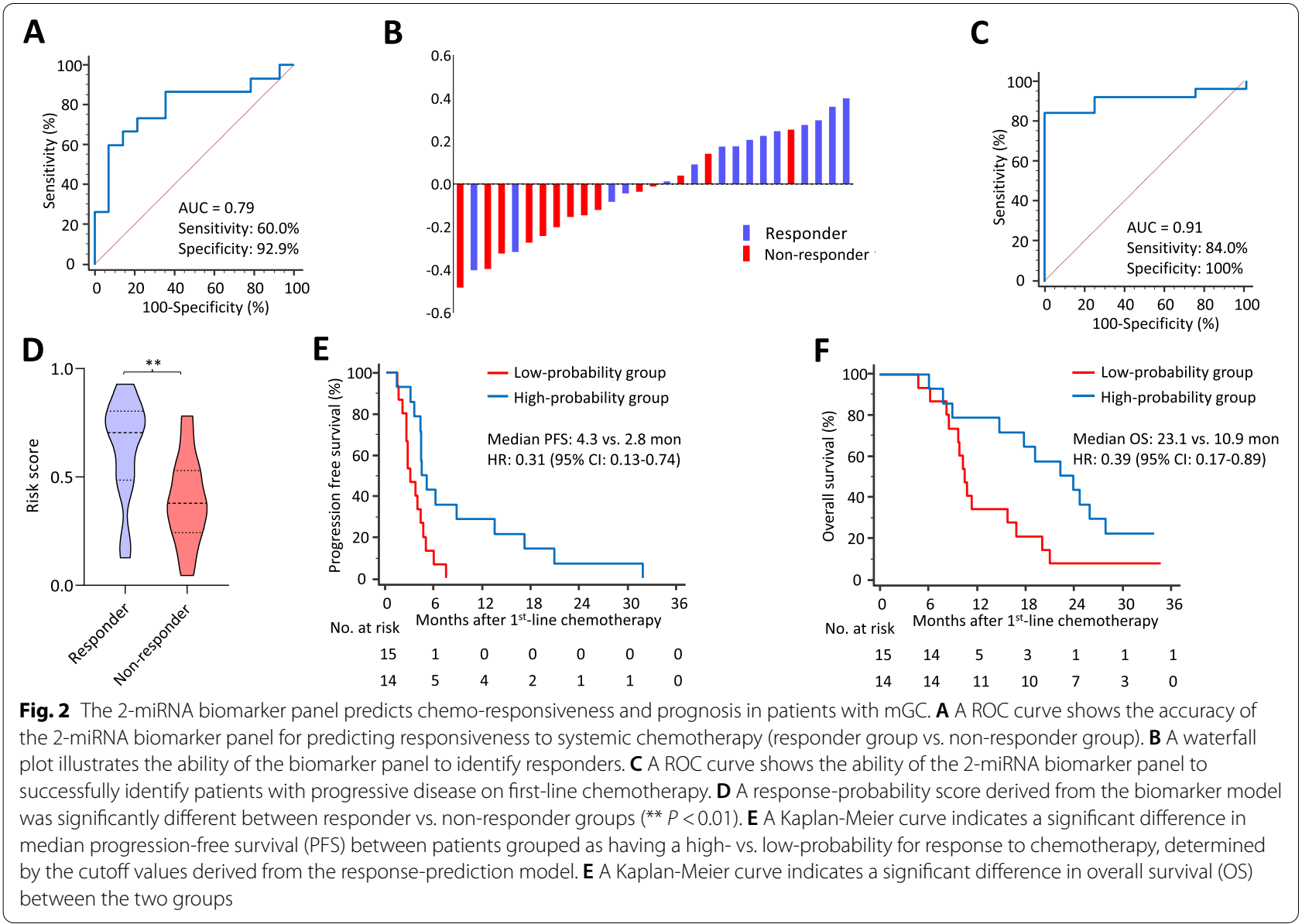


threshold values derived from the identical 2-miRNA prediction model and the proportion of responders was significantly higher in high-probability group than lowprobability group $(78.6 \%$ vs. $26.7 \%, P=0.006)$. Then we performed Kaplan-Meier analyses to determine the prognostic significance of our biomarkers. PFS in patients with a high probability of chemo-responsiveness was significantly better compared to PFS in a low probability group (median PFS: 4.3 vs. 2.8 months, $P=0.009$ ), with a hazard ratio of 0.31 (95\% CI: 0.13-0.74, $P=0.008$ ) (Fig. 2E). We also observed a significant difference in OS between two patient groups (median OS: 23.1 vs. 10.9 months, $P=0.021$ ), with a hazard ratio of 0.39 (95\% CI: $0.17-0.89, P=0.025$ ) (Fig. $2 F$ ). Other clinical variables were not associated with prognosis. These results highlight that, in addition to its ability to predict chemo-responsiveness, our biomarkers are clinically significant in stratifying the prognosis of patients with mGC.

\section{Expression of the two miRNAs is significantly upregulated upon tumor progression}

To determine whether expression of the two miRNAs changed following tumor progression, we analyzed and compared serum samples collected before initiation of first-line chemotherapy vs. upon disease progression in the clinical cohort. Upon tumor progression, we observed significant upregulation of both miR-30a-5p and miR-192-5p $(P<0.001)$.

\section{Conclusions}

Through a systematic and comprehensive discovery and validation effort, we developed a novel 2-miRNA biomarker panel that predicts chemo-responsiveness to fluoropyrimidine plus oxaliplatin therapy in patients with mGC. Our biomarkers may ultimately facilitate individualized treatment and mediate alternative cytotoxic chemotherapy strategies to improve survival outcomes in patients suffering from this malignancy.

\begin{abstract}
Abbreviations
AUC: Area under the curve; $\mathrm{Cl}$ : Confidence interval; CPM: Counts per million; GC: Gastric cancer; mGC: Metastatic or unresectable gastric cancer; OS: Overall survival; PFS: Progression-free survival; RT-qPCR: Real-time quantitative reverse transcription polymerase chain reaction; TTP: Time to progression.
\end{abstract}

\section{Acknowledgements}

We would like to thank Yuma Wada, Yasuyuki Okada, Ya Cui, and Professor Wei Li for helping with their important insights into this study.

\section{Authors' contributions}

I. Lee: Study concept and design; analysis and interpretation of data; statistical analysis; drafting of the manuscript. Z. Zhu: Analysis and interpretation of data; statistical analysis; drafting of the manuscript. J. Lee: Specimen provider; acquisition of data; critical revision of the manuscript. J. Park: Specimen provider; acquisition of data; critical revision of the manuscript. X. Wu: Management and analysis of sequencing data; drafting of the manuscript. T. Ong: Acquisition of data; Analysis and interpretation of data. S. Li: Study design; Analysis and interpretation of data; statistical analysis. X. Wang: Analysis and interpretation of data; statistical analysis; critical revision of the manuscript. J. Chao: Study concept and design; analysis and interpretation of data; drafting of the manuscript; critical revision of the manuscript. A. Goel: Study concept and design; analysis and interpretation of data; drafting of the manuscript; critical revision of the manuscript. The author(s) read and approved the final manuscript.

\section{Funding}

The present work was supported by the grants CA72851, CA181572, and CA187956 from the National Cancer Institute, National Institutes of Health, a Research award from the American Gastroenterological Association's Robert \& Sally Funderberg Research Award in Gastric Cancer, and a pilot grant award from the Stupid Strong Foundation to A. Goel. This study was also supported by National Institutes of Health Grant 5K12CA001727-23 to J. Chao. Research reported in this publication included work performed in the Integrative Genomics Core supported by the National Cancer Institute of the National Institutes of Health under award number P30CA033572. The content is solely the responsibility of the authors and does not necessarily represent the official views of the National Institutes of Health. In addition, this work was also supported by grants from the Research Grants Council of the Hong Kong Special Administrative Region, China (Project No. CityU 11102317, 11103718) and a grant from Guangdong Basic and Applied Basic Research Foundation (Project No. 2019B030302012) awarded to X. Wang.

\section{Availability of data and materials}

All data are available within the article.

\section{Declarations}

\section{Ethics approval and consent to participate}

All study-related procedures were performed as per the Declarations of Helsinki, wherein a written informed consent was obtained from each patient, and the institutional review boards of all participating institutions involved approved the study.

\section{Consent for publication}

Not applicable. The manuscript does not contain any individual personal data.

\section{Competing interests}

The authors declared that no competing interests exist.

\section{Author details}

${ }^{1}$ Department of Molecular Diagnostics and Experimental Therapeutics, Beckman Research Institute of City of Hope, Biomedical Research Center, Comprehensive Cancer Center, 1218 S. Fifth Avenue, Monrovia, CA 91016, USA. ${ }^{2}$ Department of Surgery, Asan Medical Center, University of Ulsan College of Medicine, Seoul, South Korea. ${ }^{3}$ Department of Biomedical Sciences, City University of Hong Kong, Hong Kong, China. ${ }^{4}$ Division of Hematology-Oncology, Department of Medicine, Samsung Medical Center, Sungkyunkwan University School of Medicine, Seoul, South Korea. ${ }^{5}$ Integrative Genomics Core, Beckman Research Institute of City of Hope, Duarte, CA, USA. ${ }^{6}$ Department of Medical Oncology and Therapeutics Research, City of Hope Comprehensive Cancer Center, 1500 E. Duarte Road, Duarte, CA 91010, USA. ${ }^{7}$ City of Hope Comprehensive Cancer Center, Duarte, CA, USA.

Received: 8 October 2021 Accepted: 19 December 2021 Published online: 03 January 2022

\section{References}

1. Bouche O, Raoul JL, Bonnetain F, Giovannini M, Etienne PL, Lledo G, et al. Randomized multicenter phase II trial of a biweekly regimen of fluorouracil and leucovorin (LV5FU2), LV5FU2 plus cisplatin, or LV5FU2 plus irinotecan in patients with previously untreated metastatic gastric cancer: a Federation Francophone de Cancerologie Digestive Group Study--FFCD 9803. J Clin Oncol. 2004:22:4319-28. 
2. Glimelius B, Ekstrom K, Hoffman K, Graf W, Sjoden PO, Haglund U, et al. Randomized comparison between chemotherapy plus best supportive care with best supportive care in advanced gastric cancer. Ann Oncol. 1997:8:163-8.

3. Ajani JA, D'Amico TA, Almhanna K, Bentrem DJ, Chao J, Das P, et al. Gastric Cancer, version 3.2016, NCCN clinical practice guidelines in oncology. J Natl Compr Cancer Netw. 2016;14:1286-312.

4. Guideline Committee of the Korean Gastric Cancer Association DWG, Review P. Korean practice guideline for gastric cancer 2018: an evidencebased, multi-disciplinary approach. J Gastric Cancer. 2019;19(1):1-48.

5. Japanese Gastric Cancer A. Japanese gastric cancer treatment guidelines 2018 (5th edition). Gastric Cancer. 2021;24(1):1-21.

6. Smyth EC, Verheij M, Allum W, Cunningham D, Cervantes A, Arnold D, et al. Gastric cancer: ESMO clinical practice guidelines for diagnosis, treatment and follow-up. Ann Oncol. 2016;27:v38-49.

7. Cheong JH, Yang HK, Kim H, Kim WH, Kim YW, Kook MC, et al. Predictive test for chemotherapy response in resectable gastric cancer: a multicohort, retrospective analysis. Lancet Oncol. 2018;19:629-38.

8. Kubota Y, Kawazoe A, Sasaki A, Mishima S, Sawada K, Nakamura Y, et al. The impact of molecular subtype on efficacy of chemotherapy and checkpoint inhibition in advanced gastric cancer. Clin Cancer Res. 2020;26:3784-90.

9. Schwartz LH, Litiere S, de Vries E, Ford R, Gwyther S, Mandrekar S, et al. RECIST 1.1-update and clarification: from the RECIST committee. Eur J Cancer. 2016;62:132-7.

\section{Publisher's Note}

Springer Nature remains neutral with regard to jurisdictional claims in published maps and institutional affiliations.

- fast, convenient online submission

- thorough peer review by experienced researchers in your field

- rapid publication on acceptance

- support for research data, including large and complex data types

- gold Open Access which fosters wider collaboration and increased citations

- maximum visibility for your research: over 100M website views per year

At BMC, research is always in progress.

Learn more biomedcentral.com/submissions 